RASĀYAN J. Chem.

Vol. 13 | No. 1 |481 - 487| January - March | 2020 ISSN: 0974-1496 | e-ISSN: 0976-0083 | CODEN: RJCABP

\title{
WETTING ABILITY OF A PHYTOPREPARATION AND THEIR ASSOCIATES WITH POLYELECTROLYTES
}

\author{
O. Yessimova ${ }^{1}$, S. Kumargaliyeva ${ }^{1}$, M. Kerimkulova ${ }^{1}$, K. Mussabekov ${ }^{1}$ \\ and Zh. Toktarbay, ${ }^{1,2}$ \\ ${ }^{1}$ Department of Analytical, Colloidal Chemistry and Technology of Rare Elements, Faculty of \\ Chemistry and Chemical Technologies, Al-Farabi Kazakh National University, Al-Farabi avenue \\ 71, Almaty, 050012, Kazakhstan. \\ ${ }^{2}$ Specialized gymnasium for gifted children teaching in three languages named after Al-Farabi, \\ Khabiblin Street 9, Almalybak, Almaty obl., 040909, Kazakhstan. \\ *E-mail: zhexenbek.toktarbay@gmail.com
}

\begin{abstract}
In this work, the wetting ability of the VK phytopreparation and its associates with polyelectrolytes were studied. VK is a complex of biologically active substances (BAS) isolated from Alhagi (family - Fabacae Lindi). The association of a phytopreparation with Polyhexamethylene guanidine hydrochloride (PHMG) occurs due to hydrogen bonds stabilized by hydrophobic interactions of non-polar groups of a polyelectrolyte. Therefore, intensive compaction of macromolecules of the phytopreparation-PHMG complex takes place and the viscosity of the mixture of solutions is greatly reduced. In this case, the surface of the complex will be enriched (saturated) with polar groups, which causes a decrease in the contact angles of solutions of associates compared with the phytopreparation, as well as the fraction of the occupied surface. PHMG and phytopreparation have a weak hydrophilizing effect on the surface of Teflon. The composition of the reagents under consideration suggests a certain imbalance in the hydrophilic and lipophilic properties of macromolecules towards the polar part. The low values of the surface fraction and their associates show that a sufficiently rarefied adsorption layer forms on the surface of quartz and Teflon.
\end{abstract}

Keywords: Polyhexamethylene Guanidine Hydrochloride, Polyelectrolyte, Phytopreparation, Adsorption, Wetting

(C) RASĀYAN. All rights reserved

\section{INTRODUCTION}

Interpolymer complexes as new surfactants have a great theoretical and practical interest. The changes in the surface-active properties of macromolecules during complex formation and the influence of various factors make it possible to purposefully control their properties and obtain substances with the necessary set of practically important characteristics.

Despite the study of polymer adsorption on layers theoretically and practically, this question still attracts the attention of most researchers. ${ }^{1}$ The works on the topography and density of the polymer layer, the behavior of polyelectrolytes (PE) on charged and uncharged surfaces are the basis of modern research. In addition, the significant effect of surfactants on the adsorption layers of polymers, on the properties of systems stabilized by these PEs, and, in the end, on their practical application is estimated. ${ }^{2}$

The approach to optimizing the composition of surfactant mixtures, based on the results of measuring surface tension and wetting was successfully implemented in the works of many authors. ${ }^{3,4}$ The relationship between the wetting effect, degree of hydroxyethylation and surface energy characteristics was studied in aqueous solutions of hydroxyethylated derivatives of higher fatty alcohols, imides and isononylphenols. ${ }^{5}$

The surface-active properties play an important role in the practical use of the VK phytopreparation and its associates with metacid and DDS. This is an important factor in evaluating the wetting ability of

Rasayan J. Chem., 13(1), 481-487(2020)

http://dx.doi.org/10.31788/RJC.2020.1315566

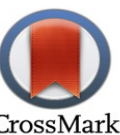


RASĀYAN J. Chem.

Vol. 13 | No. 1 | 481 - 487| January - March | 2020

aqueous solutions of these substances. As it is well known, the wettability of a solid surface by liquids is determined by Young equation; the value of the contact angle $(\theta)$.

$$
\gamma_{S V}-\gamma_{S L}=\gamma_{L V} \cos \theta
$$

WEhere, $\gamma_{\mathrm{SV}}, \gamma_{\mathrm{SL}}, \gamma_{\mathrm{LV}}$ are the interfacial tension of solid-air, solid-liquid, and liquid-air. $\theta$ is the contact angle in Young equation. According to the above equation, the smaller value of $\gamma_{\mathrm{SV}}$ and $\gamma_{\mathrm{LV}}$ is revealed at the greater value $\cos \theta$. And this value can be adjusted using a surfactant. Thus, the effectiveness of the phytopreparation VK and its associates as fungicidal, bactericidal and biological activity are determined by the wetting ability of their aqueous solutions on the hydrophobic surfaces of leaves and plant stems. In this regard, we studied the kinetics of reducing the surface tension of aqueous solutions of these substances. ${ }^{6}$ With the increase of concentration, the surfactant molecular adsorbed on the monolayer further arranged into bilayer structures by hydrophobic interaction, leading to the decrease of contact angle. ${ }^{7}$ Khan et.al studied the interaction between water-soluble polymers (polyacrylamide, partially hydrolyzed polyacrylamide, xanthan gum) and anionic surfactants such as Sodium dodecyl sulfate (SDS) and sodium dodecylbenzene sulfonate (SDBS) has been studied. ${ }^{8}$

In the works ${ }^{9,10}$ authors synthesized a series of surfactants and investigated their wettability on surfaces. They found that cationic surfactant could adsorb on the quartz surface via electrostatic interaction and form monolayer within a certain surfactant concentration range, resulting in the increase of contact angle. With the increase of concentration, the surfactant molecular adsorbed on the monolayer further arranged into bilayer structures by hydrophobic interaction, leading to a decrease of contact angle. Joanna et. al. ${ }^{11}$ also investigated the wettability of polymers and polymer-surfactant interactions. The wettability of synthetic surfactant, polymer surfactant, and composition of polymer-surfactants were studied in the existing researches. However, the wettability of biologically active substances and polymer composition has not been widely studied yet.

\section{Materials and Methods}

\section{EXPERIMENTAL}

Polyhexamethylene guanidine hydrochloride (PHMG) purchased from Pokrovsky (Russian Federation), was used as a polyelectrolyte. Phytopreparation (VK) is a complex of biologically active substances (BAS), isolated from Alhagi (Fabaceae Lindi family), prepared in our laboratory [12]. Distilled water was used as a solvent in the experiment.

The viscosity of the solution was determined by Ubbelohte viscosimetry. Optical density was measured at $298 \mathrm{~K}$ by spectrophotometer LEKI SS 1103 (Finland). The contact angle was determined by contact angle goniometer LK-1 (Russia). Wilhelmy plate method was used to measure the interfacial tension.

\section{RESULTS AND DISCUSSION}

The wetting isotherms on the surface of quartz and Teflon with aqueous solutions of metacide, VK phytopreparation, and their associates are shown in Figs.-1 and 2. All reagents used have a high molecular weight structure and the equilibrium contact angle is determined by the kinetics of formation of the polymer adsorption layer at the phase boundaries. This process is determined by the rate of diffusion of macromolecules to the interphase boundary and the surface layer in accordance with Rebinder's rule of polarity equalization. ${ }^{13}$

It was found (Fig.-1 and Fig.-2) that the main characteristics of the interfacial layers undergo noticeable changes in the first 10-15 min. It requires 24-28 hours to achieve complete adsorption equilibrium depending on the molecular weight of the polymers and the concentration of their solutions. After 15-20 minutes, the changes in $\theta$ values are very small, they can be neglected. In this regard, in our experiments, the contact angle was measured 10-15 minutes after applying the drop to a solid surface. It can be seen that the reagents have a relatively low hydrophobic effect on the surface of quartz and the contact angles increase from $5^{\circ}$ to $20-40^{\circ}$. However, in comparison with synthetic polyelectrolytes, polyacrylic, polymethacrylic acids $(\theta=2-8) \cdot{ }^{14,15}$ hydrophobic effect in the process of wetting is much higher. ${ }^{16}$

In contrast to these surfactants, the diphilicity of the VK phytopreparation is apparently due to the presence of a wide variety of functional groups: - $\mathrm{OH},-\mathrm{COOH},-\mathrm{NH},-\mathrm{NH}_{2}$ and hydrophobic groups. This should also give significant surface activity to the phytopreparation VK. This effect may be 
RASĀYAN J. Chem.

Vol. 13 | No. 1 | 481 - 487| January - March | 2020

associated with the adsorption of PHMG, a phytopreparation, and their associates on the surface of quartz as a result of the occurrence of hydrogen bonds between the functional groups of polymers $(-\mathrm{OH},-\mathrm{N}, \mathrm{H},-$ $\mathrm{COOH})$ and the surface of quartz $(-\mathrm{SiOH})$.

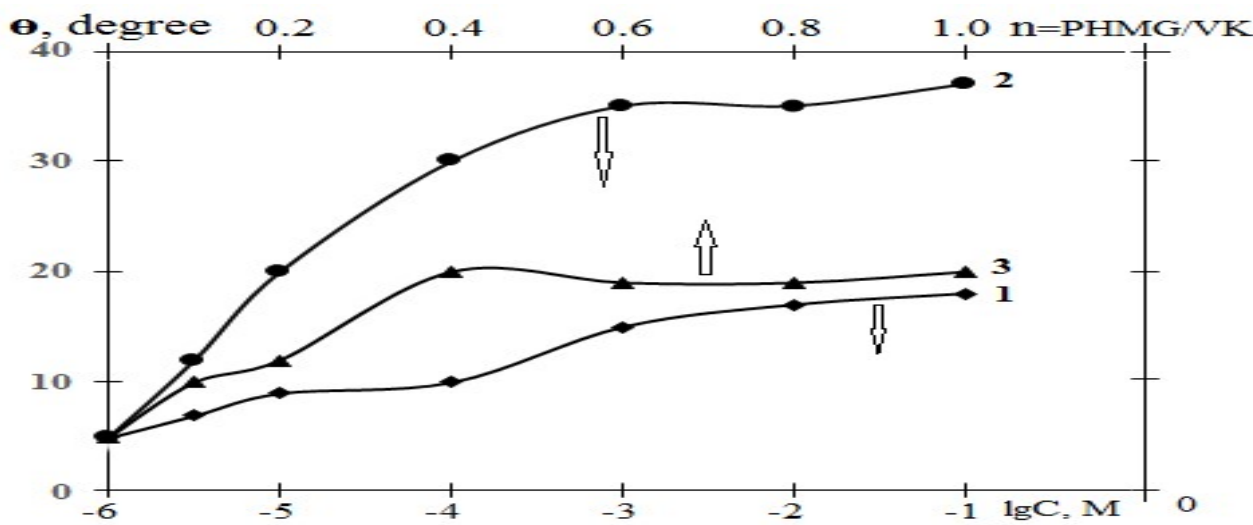

Fig.-1: Wetting Isotherms of Aqueous Solutions of PHMG (1), Phytopreparation (2) and Associates of PHMG/VK (3) on Quartz Surfaces

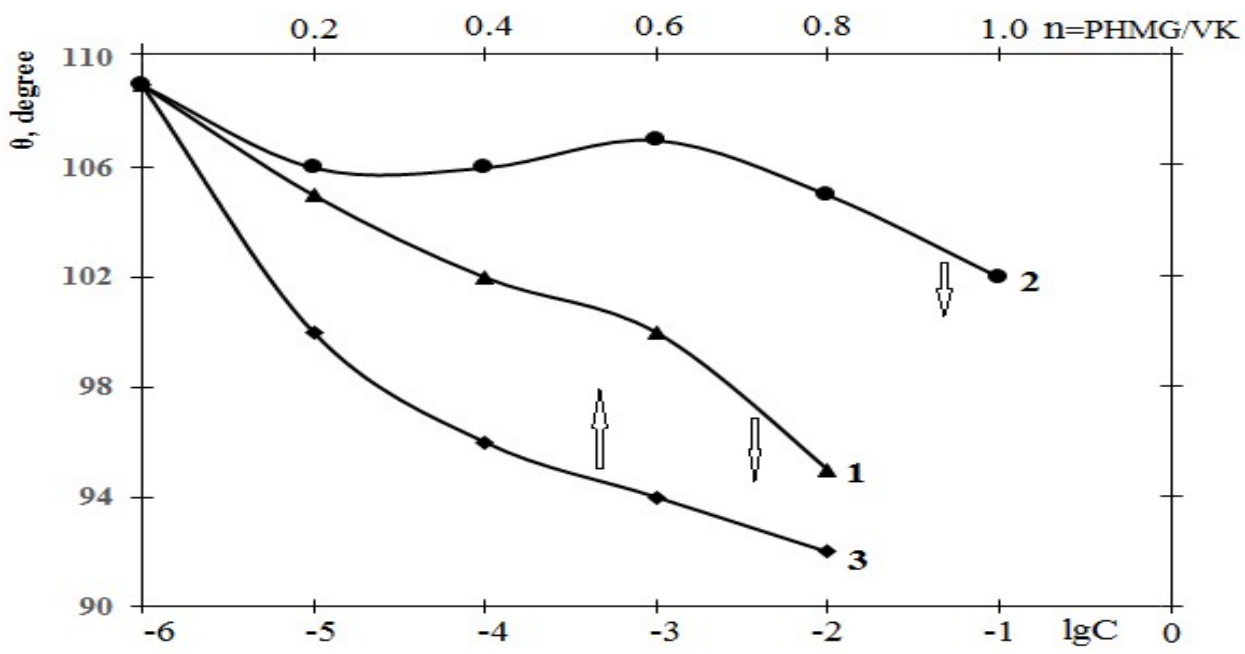

Fig.-2: Wetting Isotherms of Aqueous Solutions of PHMG (1), Phytopreparation (2) and Associates of PHMG/VK (3) on Teflon Surfaces

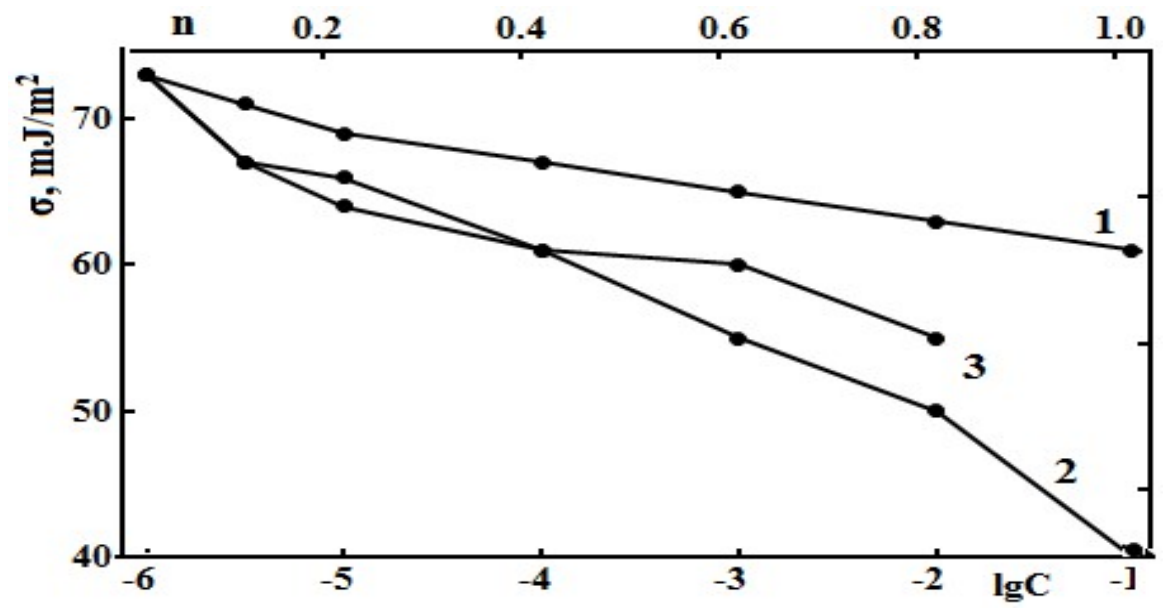

Fig.-3: Surface Tension Isotherm of PHMG (1)Phytopreparation (2) and their Complex (3) 
RASĀYAN J. Chem.

Vol. 13 | No. 1 |481 - 487| January - March | 2020

The least wetting effect is shown by PHMG, for which, as for all polyelectrolytes, the process of formation of the adsorption layer at the phase boundaries flows slowly. For metacid, a slight decrease in surface tension is also observed (Fig.-3, curve 1).

A stronger hydrophobizing effect of the phytopreparation is associated with the presence of low molecular weight amino acids in its composition. The rate and amount of adsorption of these amino acids are much higher in comparison with the polymer components (polysaccharides-43\%) of the phytopreparation. ${ }^{17}$ It should be noted that the presence of a large number of - OH groups in the polysaccharides also promotes their adsorption on the surface of the quartz. These factors apparently contribute to the adsorption and subsequent differentiation in the polarity of the phytopreparation molecules at the water/air interface and, accordingly, a noticeable decrease in the value (Fig. 3, curve 2).

The fraction of the estimate of the adsorption of the reagents used on the surface of solids based on the theory of wetting of heterogeneous surfaces, the fraction of the surface occupied by adsorbed molecules was calculated. The results are presented in Table-1.

With an increase in the concentration of phytopreparation solutions (range $10^{-3}-10^{-1} \%$ ), its activity at the phase boundaries noticeably increases. In this concentration range, apparently at the interface, there is a sufficient number of macromolecules and the degree of their differentiation in polarity plays a decisive role. ${ }^{18,19}$ The work of cohesion (Wc) and adhesion (Wa) to the surface of quartz (Teflon) were calculated from the equations:

$$
\begin{array}{r}
W_{c}=2 \sigma_{3}(\mathrm{a}) \\
W_{\mathrm{a}}=\sigma_{3}(1+\cos \theta)(\mathrm{b})
\end{array}
$$

The increase in the work of adhesion of the metacide and phytopreparation to the surface of the quartz turned out to be close to the work of their cohesion (Table-2).

In accordance with the Dupre equation, for $\mathrm{f}=\mathrm{W}_{\mathrm{a}}-\mathrm{W}_{\mathrm{c}}$, the spreading becomes unbounded. The process of unlimited spreading is a nonequilibrium process, it should also be taken into account that in the case of micromolecules, achieving equilibrium at the interphase boundaries takes a long time and is practically unattainable. For nonequilibrium processes, the equations of both Dupre and Young are not applicable.

Table -1: The Proportion of Surface Areas of Quartz and Teflon Occupied by Adsorbed Molecules of PHMG and

\begin{tabular}{|c|c|c|c|c|c|c|c|}
\hline \multirow[t]{3}{*}{$\mathrm{C}, \%$} & \multirow{3}{*}{$n=\frac{[V K]}{[P H M G]}$} & \multicolumn{6}{|c|}{ The Proportion of Surface Areas, $\%$} \\
\hline & & & & Phytopr & ion, VK & Complex & VK/PHMG \\
\hline & & Quartz & Teflon & Quartz & Teflon & Quartz & Teflon \\
\hline $10^{-5}$ & 0.1 & 1.2 & 5.2 & 4.6 & 4.5 & 1.2 & 3.0 \\
\hline $10^{-4}$ & 0.2 & 1.4 & 8.3 & 5.2 & 1.4 & 1.4 & 10.6 \\
\hline $10^{-3}$ & 0.4 & 3.0 & 11.3 & 4.5 & 4.6 & 4.6 & 16.9 \\
\hline $10^{-2}$ & 0.6 & 3.8 & 14.4 & 8.3 & 5.1 & 5.1 & 18.3 \\
\hline $10^{-1}$ & 0.8 & 4.6 & 8.3 & 10.6 & 5.1 & 5.1 & 21.0 \\
\hline
\end{tabular}
Phytopreparation VK

Table-2: The Work of Cohesion (Wc) and Adhesion (Wa) of Phytoprepration (VK), PHMG and their Complexes

\begin{tabular}{|c|c|c|c|c|c|c|c|c|c|c|}
\hline \multirow[t]{4}{*}{$\mathrm{C}, \%$} & \multirow[t]{4}{*}{$\mathrm{n}$} & \multicolumn{9}{|c|}{ The Work of Cohesion and Adhesion, $\mathrm{mJ} / \mathrm{m}^{2}$} \\
\hline & & \multicolumn{3}{|c|}{ PHMG } & \multicolumn{3}{|c|}{ Phytoprepration VK } & \multicolumn{3}{|c|}{ Complex VK:PHMG } \\
\hline & & \multirow[t]{2}{*}{$\overline{W_{\mathrm{c}}}$} & \multicolumn{2}{|c|}{$W_{\mathrm{a}}$} & \multirow[t]{2}{*}{$W_{\mathrm{c}}$} & \multicolumn{2}{|c|}{$W_{\mathrm{a}}$} & \multirow[t]{2}{*}{$W_{\mathrm{c}}$} & \multicolumn{2}{|c|}{$W_{\mathrm{a}}$} \\
\hline & & & Quartz & Teflon & & Quartz & Teflon & & Quartz & Teflon \\
\hline $10^{-5}$ & 0.1 & 138 & 137 & 52 & 129 & 125 & 48 & 133 & 132 & 48 \\
\hline $10^{-4}$ & 0.2 & 136 & 135 & 54 & 120 & 110 & 46 & 127 & 126 & 53 \\
\hline $10^{-3}$ & 0.4 & 131 & 129 & 55 & 113 & 104 & 42 & 123 & 119 & 56 \\
\hline $10^{-2}$ & 0.6 & 126 & 123 & 56 & 98 & 89 & 39 & 120 & 116 & 56 \\
\hline $10^{-1}$ & 0.8 & 114 & 111 & 46 & 70 & 63 & 29 & 102 & 99 & 50 \\
\hline
\end{tabular}
(VK / PHMG)

It has been found that the introduction of a phytopreparation VK into an aqueous solution of PHMG leads to a significant decrease in the reduced viscosity of the solution. This process is accompanied by a significant increase (in the region of $\mathrm{n} \sim 1.0-10^{-2}$ ) of the optical density of the system. 
RASĀYAN J. Chem.

Vol. 13 | No. 1 | 481 - 487| January - March | 2020

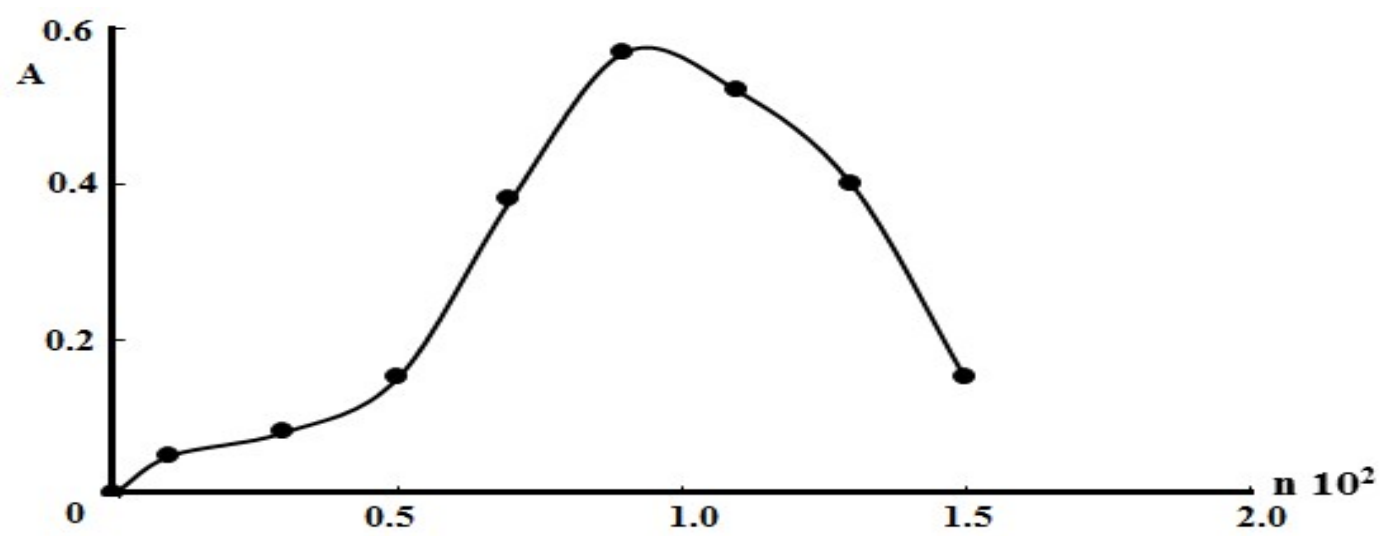

Fig.-4: The Dependence of the Optical Density of the Solution on the Mixture of PHMG with Phytopreparation VK.

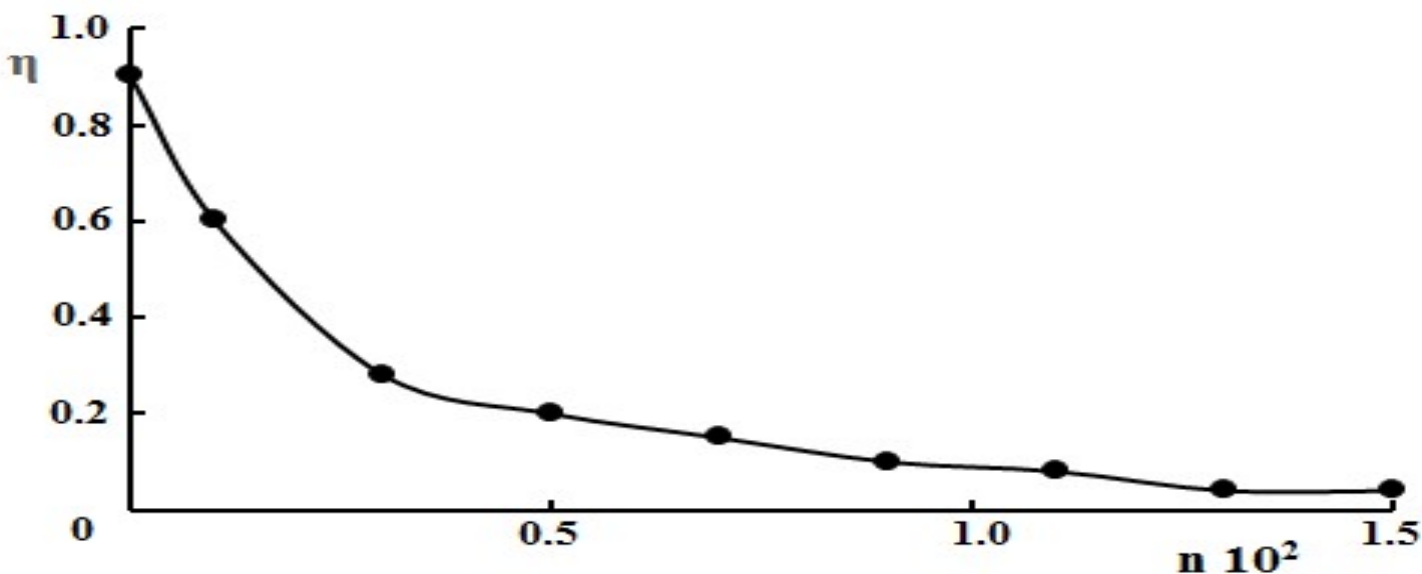

Fig.-5: Dependence of the Reduced Viscosity of the Solution on a Mixture of PHMG/VK.

It should be emphasized that the same picture is observed with reverse titration (Fig.-4 and 5). All this indicates the formation of an association between the phytopreparation VK and PHMG.

The association of a phytopreparation with PHMG occurs due to hydrogen bonds, which are stabilized by hydrophobic interaction of nonpolar groups. As a result, intensive compaction of macromolecules of the phytopreparation-PHMG complex occurs. This conclusion is confirmed by a significant decrease in the viscosity of the mixture of solutions. In this case, the surface of the coil of the complex will be enriched in polar groups. Therefore, the hydrophobizing effect decreases - the wetting angles and the fraction of the occupied surface decrease.

PHMG and phytopreparation have a weaker hydrophilizing effect than their associates on the surface of Teflon. The composition of the reagents under consideration suggests a certain imbalance in the hydrophilic and lipophilic properties of macromolecules towards the polar part. Therefore, their adsorption on the surface of Teflon deteriorates with the orientation of the polar groups in the aqueous phase. However, the fraction of the surface occupied by adsorbed macromolecules on Teflon is higher than on quartz. This indicates a greater degree of the unfolding of macromolecules on hydrophobic surfaces than on hydrophilic ones..$^{20-23}$

In accordance with this fact, during the adsorption of macromolecules on Teflon, an increase in the area occupied by one macromolecule on the surface occurs.

The work of adhesion to the Teflon surface is much smaller than the work of cohesion (Table 2). In our experiment, the wetting condition $\mathrm{Wa}>0.5 \mathrm{Wc}$ is not fulfilled for Teflon and, therefore, a slight change $\Theta$ during the wetting of Teflon with aqueous solutions of the reagents is natural.

The low values of the surface fraction and their associates show that a sufficiently rarefied adsorption layer forms on the surface of quartz and Teflon. 


\section{RASĀYAN J. Chem. \\ Vol. 13 | No. 1 |481 - 487| January - March | 2020}

Thus, the obtained results indicate the possibility of hydrophobization of lyophilic surfaces with the studied reagents. It can be assumed that their action will appreciably increase upon preliminary adsorption of these substances by prolonged exposure of solid surfaces (for 6-24 hours) in their solutions.

\section{CONCLUSION}

From the surface tension isotherms, the surface activity of the PHMG, VK and their mixture were calculated. As a result of studying, the surface properties of phytopreparation compositions with PHMG and individual components at the liquid-gas and solid-liquid interfaces, it was shown that the compositions have a high surface, adsorption and wetting activity compared to individual substances. The values of the fraction of the surface occupied by adsorbed molecules indicate the formation of a rarefied adsorption layer

It was also found that the fraction of the surface occupied by adsorbed associate molecules on Teflon is greater than on quartz. This indicates a greater degree of the unfolding of macromolecules on a hydrophobic surface, which helps to improve wetting.

The correlation between the calculated values of the work of cohesion (Wc) and adhesion (Wa) also confirms the good wetting properties of the associate PHMG/VK.

Based on the results of viscometric, spectrophotometric studies, it was found that the associates of the phytopreparation VK with polyhexamethylene guanidine hydrochloride (PHMG) are formed due to electrostatic and hydrogen bonds stabilized by hydrophobic interactions.

\section{ACKNOWLEDGMENT}

This work has been done under the financial aid of the Republic of Kazakhstan. The number of the project is AP05132247.

\section{REFERENCES}

1. P. Claesson, A. Dedinaite, O. Rojas, Advances in Colloid and Interface Science, 104, 53(2003), DOI: $10.1016 / \mathrm{s} 0001-8686(03) 00036-8$

2. P. Claesson, E. Poptoshev, E. Blomberg, A. Dedinaite, Advances in Colloid and Interface Science, 117, (3)2005, DOI:10.1016/j.cis.2004.09.008

3. H. Wang, X. Wei, Y. Dua, D. Wang, Colloids and Surfaces A: Physicochemical and Engineering Aspects, 568, 92(2019), DOI:10.1016/j.colsurfa.2019.01.062

4. Q. Zhou, B.T. Qin, J. Wang, H.T. Wang, F. Wang, Powder Technology, 326, 7(2018), DOI: $10.1016 /$ j.powtec.2017.12.00

5. S. Lee Linde Environmental Science \& Technology,33(13), 2727(2001), DOI:10.1021/es001654a

6. K.I. Omarova, K.B. Musabekov, A.O. Adilbekova, National academy of sciences of the Republic of Kazakhstan, 2, 57(2016).

7. L. Zhang, Z.L. Wang, Z.Q. Li, L. Zhang, Z.C. Xu, S. Zhao, J.Y. Yu, Langmuir, 26, 18834(2010), DOI: $10.1021 / 1 \mathrm{la} 1036822$

8. M.Y. Khan, A. Samanta, K. Ojha, A. Mandal, Asia-Pacific Journal of Chemical Engineering, 3, 579(2008), DOI:10.1002/apj.212

9. Y. Dauletov, N. Nuraje, K. Abdiyev, Zh. Toktarbay, M. Zhursumbaeva, Journal of Surfactants and Detergents, (2019), DOI:10.1002/jsde.12283

10. Ce Wang, Xu-Long Cao, Lan-Lei Guo, Zhi-Cheng Xu, Lei Zhang, Qing-Tao Gong, Lu Zhang, Sui Zhao, Colloids and Surfaces A: Physicochemical and Engineering Aspects, 509, 564(2016), DOI:10.1016/j.colsurfa.2016.09.057

11. K. Joanna, S. Katarzyna, A. Zdziennicka, B. Janczuk, International Journal of Adhesion and Adhesives, 45, 98(2013), DOI:10.1016/j.ijadhadh.2013.05.001

12. Esimova Orynkul Arykbekovna thesis of candidate of science, Department of Chemistry, Al-Farabi Kazakh National University, Almaty, Kazakhstan (2009).

13. P.A. Rebinder, Summary of a General Colloid Chemistry Course, Vol. 1, Moscow State University, Moscow, p. 230(1949). 
RASĀYAN J. Chem.

Vol. 13 | No. 1 |481 - 487| January - March | 2020

14. H. Wanga, X. Wei, Y. Dua, D. Wang, Colloids and Surfaces A: Physicochemical and Engineering Aspects, 568, 92(2019), DOI:10.1016/j.colsurfa.2019.01.062

15. K. Holmberg, B. Jonsson, B. Kronberg, B. Lindman, Surfactants and Polymers in Aqueous Solution, John Wiley \& Sons, New York, p. 286(2002).

16. Ashutosh Mishra and Rakesh Kumar Trivedi, Rasayan Journal of Chemistry, 12(2), 1011(2019), DOI: 10.31788/RJC.2019.1225073

17. D. L. Aulifa, I. K. Adnyana, Sukrasno and J. Levita, Rasayan Journal of Chemistry, 13(1), 11(2020), DOI: $10.31788 /$ RJC.2020.1315397

18. K. Omarova, M. Kerimkulova, Journal of Chemical Technology and Metallurgy, 54(3), 633(2019).

19. Cut Nuraskin, Marlina, R. Idroes, C. Soraya and Djufri, Rasayan Journal of Chemistry, 13(1), 18(2020), DOI:10.31788/RJC.2020.1315434

20. A. Zdziennicka, B. Janczuk, W. Wojcik, Journal of Colloid and Interface Science, 268, 200(2003), DOI: 10.1016/S0021-9797(03)00702-1

21. X. Zhao, Y. Gao, C. Zhang, Y. Zhu, J. Lei, Y. Ma, F. Du, Journal of Dispersion Science and Technology, (2018), DOI:10.1080/01932691.2018.1462193

22. Y. Dauletov, K. Abdiyev, Zh. Toktarbay, N. Nuraje, M. Zhursumbaeva and B. Kenzhaliyev, Fibers and Polymers, 19, 2023(2018), DOI:10.1007/s12221-018-6947-3

23. Kalyani Sreekumar, B. Bindhu, K. Veluaraja and V. L. Reena, Rasayan Journal of Chemistry,12(4), 1872(2019), DOI: 10.31788/RJC.2019.1245332

[RJC-5566/2019] 